\title{
Human endogenous retroviruses in health and disease: a symbiotic perspective
}

\author{
Frank P Ryan FRCP FLS
}

J R Soc Med 2004;97:560-565

In the early 1970 s, scientists scanning baboon placentas with the electronmicroscope were startled to discover endogenous retroviruses budding from the syncytiotrophoblast. Similar viruses were subsequently detected in the placentas of healthy cats, mice, guineapigs and humans. ${ }^{1}$ Retroviruses were originally discovered when Peyton Rous noticed the effects of the avian sarcoma virus in 1910, but their infectious and pathogenic potential for humans only became clear with the discoveries of the sexually transmitted human T-cell leukaemia viruses, HTLV-I and HTLV-II, and even more so with the arrival of the human immunodeficiency viruses, notably HIV-1, which ushered in the lethal pandemic now underway.

The genomes of retroviruses are based on RNA. To insert themselves into the DNA-based genome of humans they possess a unique enzyme, reverse transcriptase (RT), that copies the viral RNA template to its complementary DNA, which is then integrated into the chromosomes. Retroviruses have two very different life strategies. Exogenous retroviruses, like HIV, reproduce within the somatic tissues of infected individuals and spread in the human population through sexual intercourse and contaminated blood products as well as from mother to child by passage of virus transplacentally or during parturition, or via breast milk. Although it is likely that the HTLV and HIV viruses first infected humans through crossspecies spread from animal hosts, their natural host is now the human species. Endogenous retroviruses (ERVs) have invaded the germ cell lines of every species of vertebrate. Here they replicate in Mendelian fashion, as an integral part of the sexual reproduction of the host, to inhabit the genome of all future generations. This is known as germline transmission. It has important evolutionary, physiological and pathological implications.

Genome sequencing reveals that $8 \%$ of the human genome consists of human endogenous retroviruses, or HERVs, and, if we extend this to HERV fragments and derivatives, the retroviral legacy amounts to roughly half our DNA.2,3 Although many HERVs have been degraded into fragments, they are still readily identifiable from the

Southwest Primary Care Trust, Fulwood House, 5 Old Fulwood Road, Sheffield, S10 3TH, UK

E-mail: ryanfrankp@aol.com

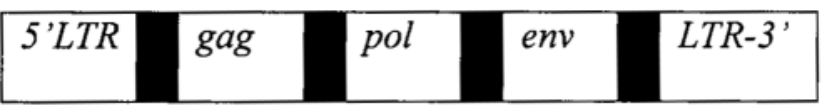

Figure 1 Geonomic structure of a human endogenous retrovirus

presence of any of three pathognomonic genes, gag, pol and env, and their flanking long terminal repeats or LTRs (see Figure 1). These genes, which they share with HTLV and HIV, are subdivided into regions with different functions. For example, the env gene codes for the surface protein of the virus as well as regions responsible for inducing human cell fusion and immunosuppression. Tens of thousands of HERVs have entirely lost their genes, leaving a legacy of vast numbers of solitary LTRs that still retain a variety of genetic potentials. Human endogenous retroviruses are believed to be the legacy of ancient germ cell infections by exogenous retroviruses, dating from 60 million years ago to the present. Pandemic plagues are a brutal manifestation of an evolutionary mechanism resulting in changes to the species gene pool I have labelled 'plague culling'. Assuming that the exogenous forebears of our human endogenous retroviruses once behaved as highly contagious infections, following transmission pathways akin to that of HIV-1, their potential for pandemic spread through the original African-based population of primates and subsequently hominids implies a protracted series of invasions with repeated large-scale attrition. It is salutary to realize that we modern humans are descended from the survivors of this terrible evolutionary legacy. The most recently integrated human endogenous retrovirus yet discovered is HERV-K113, found on chromosome 19 in just 29\% of people of mainly African, Asian and Polynesian extraction. It could only have been incorporated into the human genome after the last great migration from Africa, certainly less than 200000 years ago and possibly much more recently. ${ }^{4}$ Like the virus designated HERV-H/RGH-2, which is associated with multiple sclerosis, it may retain some degree of horizontal transmissibility. ${ }^{5}$ However, the great majority of HERVs have lost the capacity for horizontal transmission and have entered into a longstanding union with the rest of the human genome.

\section{HERVs in disease}

Given their interactive nature within the genome, HERVs have obvious potential for genetic disorders. Microdeletions 
of the long arm of the human $\mathrm{Y}$ chromosome can cause male infertility. They arise through non-allelic homologous recombination between HERV elements on the Y chromosome. ${ }^{6}$ Approximately $20 \%$ of cancers are attributable to exogenous viruses. For example, HTLV-I and HTLV-II cause adult T-cell leukaemia, lymphoma and hairy cell leukaemia; HIV-1 and HIV-2 are indirect causes of lymphoma and Kaposi's sarcoma; and human papillomaviruses cause cervical and penile carcinomas. ${ }^{7}$ HERVs have also been linked to the causation of human diseases, including carcinogenesis. Retrovirus-related elements massively repeated in the human genome include LTRs and the long and short interspersed repeats known as LINES and SINES. ${ }^{8}$ The fact that humans have twice as many LINES as chimpanzees suggests that large numbers have been acquired since the human line split from the presumptive common ancestor. Varieties of SINE, known as Alu elements, are restricted to primates. Consisting of roughly 300 base pairs, these 'pseudogenes' have massively replicated themselves in our ancestral genome and now number more than a million copies. Indeed, they continue to form new copies at the mean rate of one every 200 births.

The great majority of such retroposon insertions do not give rise to disease. However, Batzer and Deininger have reported Alu-induced mutations that may alter gene expression or disrupt reading frames and gene splicing, accounting for some individual or familial cases of germ-line diseases including neurofibromatosis, pseudoxanthoma elasticum, haemophilia, breast cancer, Apert's syndrome, cholinesterase deficiency and complement deficiency. ${ }^{9}$ The same workers describe Alu-induced 'ectopic recombinations' (recombination between different, or non-allelic, regions of the chromosomes) that are thought to cause some cases of insulin-resistant type 2 diabetes, LeschNyhan syndrome, Tay-Sachs disease, complement component C3 deficiency, familial hypercholesterolaemia and $\alpha$-thalassaemia. They estimate that $0.4 \%$ of human genetic diseases are the result of such genetic aberration brought about by a small percentage of Alu elements. They also report several types of cancer, including Ewing's sarcoma, breast cancer and acute myeloid leukaemia, as associated with Alu-mediated recombination - an association that does not as yet amount to definite aetiology. In an important overview, Nelson and colleagues have further outlined the potential of HERVs and other of their products to influence disease through genetic mutation, modulation and undesired expression of products. ${ }^{10}$ LTRs may act as biological transducers and HERV expression may itself be altered by signals, whether hormonal or through interactions with common acute viruses. For example, cytomegalovirus and the Epstein-Barr virus have been shown to transactivate HERV-K. There is even the possibility of expressive interaction with exogenous retroviruses, such as HIV-1. Other cancers linked to HERVs include seminomas, testicular teratocarcinomas and choriocarcinomas as well as certain breast cancers, small-cell lung carcinomas, renal cancers and leukaemias. Of these, the germ-cell derived tumours have received particular attention, with much circumstantial though as yet inconclusive evidence. ${ }^{11-13}$ So far nobody has been able to prove a definite aetiological role for HERVs in these cancerous conditions, but Nelson feels that the sum weight of evidence makes a convincing case for what will probably turn out to be a variety of complex and interactive mechanisms. LINE 1 insertions are associated with some cases of muscular dystrophy and haemophilia. Another major disease group associated with HERV expression are the autoimmune diseases including rheumatic disorders, systemic lupus erythematosus, multiple sclerosis, type I diabetes, alopecia areata, Sjögren's syndrome, primary biliary cirrhosis and systemic sclerosis. ${ }^{14}$ Once again, however, to date none of these associations has been definitively proven as causative to the disease.

While some of these associations may well play a part in aetiology, we need to bear in mind that HERVs are not acute viruses, behaving selfishly. Indeed there is growing evidence that HERVs are symbiotic partners that have been integrated into the human genome for millions of years. As Johnson and colleagues warn us, their expression might be augmented or disturbed as a secondary phenomenon in cancerous tissues or tissues involved in inflammatory responses, sometimes making them tumour markers or even a normal aspect of the inflammatory process. ${ }^{15}$ Researchers have of necessity become increasingly cautious in planning and interpreting their studies.

\section{HERVs in physiology}

Concurrent with these investigations of HERVs in disease, a very different line of inquiry has shed a startling new light on the relation of HERVs to human evolution and physiology. In 2001, O’Neill and her colleagues showed how retroviruses inhabiting the centromeres of the chromosomes of hybrid Australian rock wallabies are creating new species by wholesale juggling of chromosomal fragments. ${ }^{16}$ In that same year Hughes and Coffin used phylogenetic and sequence analysis to suggest that human endogenous retroviruses may have induced large-scale deletions, duplications and chromosome reshuffling in human genomic evolution. ${ }^{17}$ In the opinion of the geneticist Eugene Sverdlov, these viruses played a significant role in the evolution and divergence of the hominids. ${ }^{18}$

This line of discovery gained momentum in the 1980s when virologists, among them Erik Larsson at the University of Uppsala, suggested that HERVs may help 
protect the fetus. Certain viruses have the capacity to fuse mammalian cells into confluent multinucleated sheets of tissue. Multinucleated giant cells are a pathological feature of AIDS. Fusion of cells is also a characteristic feature of the mammalian placenta, where a microscopically thin and confluent tissue layer known as the syncytium forms the physiological barrier between maternal and fetal circulations. All nutrients from the mother and waste from the fetus must pass through this syncytial layer. The syncytium also serves to prevent maternal immune rejection of the fetus, which inherits half of its antigens from the father. There is growing evidence that endogenous retroviruses contribute importantly to the structure and function of the syncytium.

Luis Villarreal, director of the Center for Virus Research at the University of California at Irvine, takes a special interest in the evolutionary interactions between viruses and their hosts. When Villarreal inserted a virus known to suppress endogenous retroviral genes into cells that form the placenta in mice, the placentas did not become implanted into the uterus. ${ }^{8}$ Important contributions were made by Leyden and Rote in Ohio and Johnson in Liverpool. ${ }^{19}$ These workers characterized and defined HERVs in normal human placentas, while O'Connell and colleagues went on to identify and characterize a particular virus now known as ERV-3, whose envelope gene was expressed in the syncytiotrophoblast. ${ }^{20}$ They also located ERV-3 to human chromosome 7. Continuing investigation has suggested that ERV-3 participates in the differentiation of the cytotrophoblast. ${ }^{21,22}$ Some authorities believe that the envelope gene (env) may have immunosuppressive properties that could operate in the placenta, but this is disputed and awaits confirmation. The notion of a singular role for ERV-3 in placentation received a setback when, in 1998, de Parseval and Heidmann found that about 1\% of Caucasians are homozygous for a stop codon that precludes the fusion and putative immunosuppressive functions of ERV-3 - yet pregnancy is not prevented. ${ }^{23}$ While ERV-3 is still believed to have a substantial role in placentation, it cannot be an exclusive one.

The year 2000 saw an important advance when McCoy and colleagues in America and Mallet and colleagues in France independently identified an endogenous retrovirus of the HERV-W family that was expressed almost exclusively in the placenta. ${ }^{24,25}$ This HERV was also located on human chromosome 7 and its envelope gene coded for a protein they labelled 'syncytin'. Syncytin was shown to fuse mammalian cells. Over the succeeding years, continuing investigation by the McCoy and Mallet groups, and several others, confirmed and extended this finding, and today there is general acceptance that this HERV-W and its translated product syncytin have important functions in human placental physiology. ${ }^{26}$ Other researchers raised the possibility that HERVs might help protect their hosts, including the fetuses of HIV infected mothers, by blocking infection by exogenous retroviruses. In 2003, Ponferrada and colleagues reported confirmation that the envelope glycoprotein of HERV-W protected a human tissue cell line from infection with the exogenous retrovirus known as spleen necrosis virus. ${ }^{27}$ An exciting line of investigation by Stoye and colleagues at Mill Hill suggested that a retroviral capsid protein, Fv1, might reduce the infectivity of HIV-1 in monkeys but Sodroski, of Harvard, then showed the protective agent to be a protein coded by the Trim5 gene. ${ }^{28}$

In 2003 a study involving genomewide screening for fusogenic viral envelopes resulted in the discovery of a second syncytin protein - syncytin $2 .{ }^{29}$ Confirmed to be a physiological component of the human placenta, syncytin 2 is coded by the envelope gene of the family HERV-FRD. It has a putative range of fusion and immunosuppression similar to that of syncytin though it seems to use a different cell receptor. Recently, Rote and colleagues have overviewed the functional roles of all three endogenous retroviruses, ERV-3, HERV-W and HERV-FRD, to construct a testable model of villous cytotrophoblast differentiation. Refuting any fusogenic role for ERV-3 env, they propose that its main action is hormonal, initiating the production of human gonadotropic hormone. If true, this is yet another example of a key HERV role in human physiology, suggesting that the three envelope proteins, syncytin, syncytin 2 and ERV-3 env, have differing but essentially complementary functions in the multistep process of placentation. ${ }^{30}$

HERVs have been found to contribute physiologically in other human tissues. In particular the vast numbers of residual LTRs contain regulatory elements known as promoters, enhancers, silencers and polyadenylation signals that can specifically interact with the cellular expression of proteins. HERV-derived promoters have been found in roughly a quarter of all the human promoter regions so far examined. In many such instances, the viral promoter has only a minor function. However, in 2003, Dunn and colleagues demonstrated that an LTR of the ERV-L family is the dominant promoter of the gene responsible for the enzyme galactosyltransferase in the human colon and small intestine. ${ }^{31}$ Andersson and her colleagues have produced clear evidence for developmental and physiological roles for HERV-R (also known as ERV-3), showing that it is highly expressed in many human fetal tissues including adrenal cortex, kidney tubules, tongue, heart, liver and central nervous system as well as in the sebaceous glands of normal skin. ${ }^{32,33}$ In the developing embryo, organ-specific expression of HERV-R suggests participation in tissue development and differentiation. It would appear that even the Alu elements, some of which cause genetic diseases, 
may have contributed importantly to human evolution, influencing genomic architecture, possibly stimulating protein translation by inhibiting an RNA-dependent protein kinase, PKR, and responding in a way that has yet to be fully determined to cellular stresses, for example by aiding the healing of chromosomal breaks. ${ }^{9}$ Alu elements may contribute physiologically to gene expression, acting as global modifiers of gene expression through changes in their own methylation status. ${ }^{9,34}$ Endogenous retroviruses appear to be an important and integral part of us, so we can confidently look forward to the discovery of many other roles for their genes and derivatives in human evolution, fetal development and physiology.

\section{HERVs as symbionts}

Kwang Jeon is now a professor in the Department of Biochemistry in Tennessee and editor of the International Review of Cytology. In 1966, when he was a postgraduate studying Amoeba proteus at the State University of New York at Buffalo, his cultures of amoebae were invaded by a hitherto unknown bacterium (now labelled the x-bacterium). At first the plague seemed universally lethal. However, in the weeks that followed, a small minority of amoebae survived despite the presence of tens of thousands of bacteria permanently in their cytoplasm. Then, over the succeeding year of persistent infection, something very interesting was seen to develop. When Jeon used antibiotics to kill the cytoplasmic bacteria, the amoebae also died. If, on the other hand, he released the bacteria from their cytoplasmic confinement, the bacteria also failed to survive on culture media. The bacterium and amoeba had fused into a mutually interdependent new life form, now known as the $\mathrm{x}$-amoeba. ${ }^{35}$

This union of two very different life forms is known as symbiosis. Over decades of study, Jeon has confirmed that the symbiotic union of $\mathrm{x}$-bacterium and $A$. proteus took place at genomic level. ${ }^{36}$ Jeon's observation shows how pandemic parasitism can 'cull' a host population (i.e. species gene pool) and then coevolve with the surviving residuum, to become a mutualism. It also shows us how the evolutionary force of symbiosis, known as symbiogenesis - gave rise to mitochondria and chloroplasts, making it possible for us to breathe oxygen and for plants to capture the energy of sunlight. ${ }^{37}$ Symbiogenesis is seen to begin with the random coming together of different life forms, bringing to the union very different pre-evolved abilities. Natural selection does not initiate the symbiotic union but it certainly influences the outcome. The evolutionary biologist John Maynard Smith saw a key difference between the way natural selection works in symbiogenesis and neoDarwinian evolution. Rather than, or perhaps even in addition to, operating at individual (or single gene) level, in symbiogenesis selection acts at the level of the partnership, adapting it to a stable evolutionary strategy. ${ }^{38}$ This may help us to understand the nature of the interaction between human endogenous retroviruses and the rest of the human genome.

The integration of HERVs into the human genome was assumed to be irreversible. But in 2002 Medstrand and colleagues demonstrated that HERVs can in fact be removed, perhaps through chromosome deletions but more probably through sexual homologous recombination. ${ }^{3}$ The impressive accumulation of retroelements discovered on the $\mathrm{Y}$ chromosome supports the latter, since, as an unmatched chromosome, it is precluded from elimination of HERV elements through homologous allelic recombination. Why, then, have such vast numbers of HERVs and their fragments been retained? The explanation is likely to be multifactorial but mutualism might explain part of this enigma. In a mutualistic relationship, a virus that evolved in a detrimental way to its host would threaten the partnership, as would a host that changed in any way detrimental to the virus. Natural selection would select against these eventualities while selecting for changes that preserved and strengthened the relationship. The exogenous retrovirus that gave rise to the HERV-W endogenous family is believed to have entered the ancestral genome less than 40 million years ago. Bonnaud and his colleagues have tracked the action of natural selection on the HERV-W locus, ERVWE1 (whose env gene codes for syncytin) in chimpanzee, gorilla, orangutan and gibbon, revealing a specific genetic signature crucial to the gene's fusogenic action that has been conserved by natural selection over the tens of millions of years of primate divergence. ${ }^{39}$ This is an important demonstration. It confirms Maynard Smith's prediction of natural selection working at the level of the partnership in a mutualistic symbiosis involving HERV-W and the human genome.

Symbiogenesis would also help explain several other observed features of HERVs, including the loss, over time, of their infectious independence. Natural selection would not be expected to preserve non-contributory genes in the HERV genomes. Our symbiotically derived human mitochondria have been whittled down, over two billion years of evolution, from an original complement of three thousand or so genes to thirty-seven in the mitochondria themselves and a few hundred that have moved to play a part in the nucleus.

Symbiogenesis needed to be clarified from a symbiological standpoint before it could be applied to HERVs. In my book, Darwin's Blind Spot, I redefined it as follows:

'Symbiogenesis is evolutionary change arising from the interaction of dissimilar life forms. It takes two major forms: endosymbiosis, when the interaction is at the level of the genomes, and exosymbiosis, when the 
interaction may be behavioural or involve the sharing of metabolites, including gene-coded products.' 36

De Bary's original definition of symbiosis included parasitism-indeed mutualism commonly derives from parasitism. It is thus inevitable that all HERVs begin their evolutionary association with the human genome as parasitic symbionts. Most, if not all, have lost this parasitic potential, though a few, as we have seen, retain infectivity in a much attenuated fashion. A conundrum at present unanswerable is how many of the vast numbers of HERVs in the human genome have evolved to mutualism. The evolution of mutualistic symbiosis can be analysed on a cost-benefit basis, according to the contributing traits of the participating partners. ${ }^{40}$ What is cheap to produce for one partner is expensive or impossible for the other, and vice versa. This is what gives mutualistic symbiosis such powerful evolutionary potential. Our human cells, for example, cannot readily form syncytia. The retrovirus HERV-W joined the symbiotic partnership with the preevolved ability to offer this, and perhaps several other useful potentials. What then does the human genome offer the virus in return? The answer would appear to be immortality, though at a price. As we observed in the symbiogenetic creation of the $\mathrm{x}$-amoeba, what began as independent interacting partners are honed by natural selection into a single genomic union in which the continuing interaction is no longer at the level of individual life-forms but their contributing individual genes or genetic derivatives: the HERVs in our genome have lost the ability to survive independently, but their removal from our genome would also make us extinct.

This mutualistic interpretation has received important confirmation from an independent series of investigations by Villarreal, who has concluded, from phylogenetic analysis of viral and host lineages, that $80 \%$ of viral genes have no counterparts in the eukaryotic genetic database. This contradicts an older view that retroviruses originated from eukaryotic genomes. Viruses are enormously creative in the manufacture of new genes, which they can contribute to their hosts in symbiotic unions. If Villarreal and his colleague DeFilippis are correct, one such viral contribution to the human lineage was a crucial group of DNA polymerases. ${ }^{41}$ Genomes have evolved several 'epigenetic' mechanisms to control gene expression. For example, methylation of genetic elements is one way in which a host genome can when necessary silence viral genes during development and normal metabolism. Over the past decade, a system known as RNA interference (RNAi) has emerged as another genomic mechanism for silencing unwanted gene expression-for example, of genes involved in cancer, AIDS and hepatitis. One such RNAi, which responds to the presence of double-stranded RNA genes, has been found to be important in controlling gene expression in humans as well as many other life forms and is likely to have arisen as a defence mechanism to combat invasion by double-stranded RNA viruses. In Villarreal's opinion, this shows such striking behavioural similarities to known viral systems that it may well have derived from another viral symbiosis - though its origin is far more ancient than that of human endogenous retroviruses. The American Society for Microbiology regards this subject of viral contribution to host evolution as so important that it has commissioned Villarreal to write a book to educate the next generation of scientists. ${ }^{42}$

Of course, many HERVs may not have evolved to mutualism. Moreover, even longstanding mutualistic symbioses can be damaged or disrupted. The bleaching of coral is an example of such a disruption to a hitherto evolutionary stable strategy. So too are the illnesses and genetic disorders linked to impaired function of human mitochondria. ${ }^{36}$

In 2001 Lee and colleagues found evidence that preeclampsia is accompanied by a substantial reduction in syncytin expression and its dislocation from its normal position in the syncytial tissues, ${ }^{43}$ observations that were confirmed by Knerr and colleagues. ${ }^{44}$ Since the expression and function of syncytin are reduced by hypoxia, the disturbance seen in pre-eclampsia might be secondary to poor placental perfusion ${ }^{45}$ nevertheless, the same group suggests that pre-eclampsia may be viewed as an 'altered syncytin system'. ${ }^{46}$ That the link between syncytin and preeclampsia is still far from resolved reflects the very complex and dynamic nature of the mechanics involved in the single best understood HERV mutualism.

With the expansion of studies into the role of HERVs and their products in physiology and pathology, we can look forward to rapid progress in this extraordinary new chapter of biological and medical advance.

Acknowledgments I thank Jack Lenz, Dixie Mager, François Mallet, John M McCoy, Paul N Nelson, Jonathan Stoye, Carel von Vaupel Klein and Luis P Villarreal for their courtesy and help.

\section{REFERENCES}

1 Harris JR. Placental endogenous retrovirus (ERV): structural, functional and evolutionary significance. Bioessays 1998;20:307-16

2 Bannert N, Kurth R. Retroelements and the human genome: new perspectives on an old relation. Proc Natl Acad Sci USA (in press)

3 Medstrand $\mathrm{P}$, van de Lagemaat LN, Mager DL. Retroelement distributions in the human genome: variations associated with age and proximity to genes. Genome Res 2002;12:1483-95

4 Turner G, Barbulescu M, Su M, et al. Insertional polymorphisms of full-length endogenous retroviruses in humans. Curr Biol 2001;11:1531-5 
5 Christensen T, Pedersen L, Sorensen PD, et al. A transmissible human endogenous retrovirus. AIDS Res Hum Retroviruses 2002;18:861-6

6 Blanco P, Shlumukova M, Sargent CA, et al. Divergent outcomes of intra-chromosomal recombination on the human Y chromosome: male infertility and recurrent polymorphism. J Med Genet 2002;37:752-8

7 Weiss RA. Retroviruses and cancer. Curr Sci 2001;81:528-34

8 Villarreal LP. Can viruses make us human? Proc Am Philosoph Soc (in press)

9 Deininger PL, Batzer MA. Alu repeats and human disease. Mol Genet Metab 1999;67:183-93

10 Nelson PN, Hooley P, Roden D, et al. Human endogenous retroviruses: transposable elements with potential? Clin Exp Immunol (in press)

11 Boller K, Konig H, Sauter M, et al. Evidence that HERV-K is the endogenous retrovirus sequence that codes for the human teratocarcinomas-derived retrovirus HTDV. Virology 1993;196:349-53

12 Herbst $\mathrm{H}$, Kuhler-Obbarius $\mathrm{C}$, Lauke $\mathrm{H}$, et al. Human endogenous retrovirus (HERV)-K transcripts in gonadoblastomas and gonadoblastoma-derived germ cell tumours. Virchows Arch 1999; 434:11-15

13 Boese A, Sauter M, Galli U, et al. Human endogenous retrovirus protein cORF supports cell transformation and associates with promyelocytic leukaemia zinc finger protein. Oncogene 2000;19:4328-36

14 Portis JL. Perspectives on the role of endogenous human retroviruses in autoimmune diseases. Virology 2002;296:1-5

15 Johnson JB, Silva C, Holden J, et al. Monocyte activation and differentiation augment human endogenous retrovirus expression: implications for inflammatory brain diseases. Ann Neurol 2001;50: 434 42

16 O'Neill RJW, Eldridge MDB, Graves JAM. Chromosome hererozygosity and de novo chromosome rearrangements in mammalian interspecies hybrids. Mammalian Genome 2001;12:256-9

17 Hughes JF, Coffin JM. Evidence for genomic rearrangements mediated by human endogenous retroviruses during primate evolution. Nat Genet 2001;29:487-9

18 Sverdlov ED. Retroviruses and human evolution. BioEssays 2000;22:161-71

19 Lyden TW, Johnson PM, Mwenda JM, et al. Ultrastructural characterisation of endogenous retroviral particles isolated from normal human placentas. Biol Reprod 1994;51:152-7

20 O'Connell CD, O'Brien SJ, Nash WG, et al. ERV-3, a full-length human endogenous provirus: chromosomal localisation and evolutionary relationships. Virology 1984;138:225-35

21 Venables PJW, Brookes SM, Griffiths D, et al. Abundance of an endogenous retroviral envelope protein in placental trophoblast suggests a biological function. Virology 1995;211:589-92

22 Muir A, Lever A, Moffett A. Expression and functions of human endogenous retroviruses in the placenta: an update. Placenta 2004;25(suppl A):S16-25

23 De Parseval N, Heidmann T. Physiological knockout of the envelope gene of the single-copy ERV-3 human endogenous retrovirus in a fraction of the Caucasian population. J Virol 1998;72:3442-5

24 Mi S, Lee X, Li X, et al. Syncytin is a captive retroviral envelope protein involved in human placental morphogenesis. Nature 2000;403:785-9

25 Blond J-L, Lavillette D, Cheynet V, et al. An envelope glycoprotein of the human endogenous retrovirus HERV-W is expressed in the human placenta and fuses cells expressing the type D mammalian retrovirus receptor. J Virol 2000;74:3321-9

26 Mallet F, Bouton O, Prudhomme S, et al. The endogenous retroviral locus ERVWE1 is a bona fide gene involved in hominoid placental physiology. Proc Natl Acad Sci USA 2004;101:1731-6
27 Ponferrada VG, Mauck BS, Wooley DP. The envelope glycoprotein of human endogenous retrovirus HERV-W induces cellular resistance to spleen necrosis virus. Arch Virol 2003;148:659-75

28 Stremlau M, Owens CM, Perron MJ, et al. The cytoplasmic body component TRIM5alpha restricts HIV-1 infection in old world monkeys. Nature 2004;427:848-53

29 Blaise S, de Parseval N, Bénit L, et al. Genomewide screening for fusogenic human endogenous retrovirus envelopes identifies syncytin 2, a gene conserved on primate evolution. Proc Natl Acad Sci 2003; 100:13013-18

30 Rote NS, Chakrabarti S, Stetzer BP. The role of human endogenous retroviruses in trophoblast differentiation and placental development. Placenta (in press)

31 Dunn CA, Medstrand P, Mager DL. An endogenous retroviral long terminal repeat is the dominant promoter for human $\alpha 1,3$ galactosyltransferase 5 in the colon. Proc Natl Acad Sci 2003;100: 12841-6

32 Andersson A-C, Venables PJW, Tönjes RR, et al. Developmental expression of HERV-R (ERV-3) and HERV-K in human tissue. Virology 2002;297:220-5

33 Andersson A-C, Merza M, Venables P, et al. Elevated levels of the endogenous retrovirus ERV3 in human sebaceous glands. J Invest Dermatol 1996;106:125-8

34 Batzer MA, Deininger PL. Alu repeats and human genomic diversity. Nat Rev/Genet 2002;3:370-80

35 Jeon KW. Amoeba and x-bacteria: symbiont acquisition and possible species change. In: Margulis L, Fester R, eds. Symbiosis as a Source of Evolutionary Innovation. Cambridge, Mass: MIT Press, 1991

36 Ryan F. Darwin's Blind Spot: Evolution Beyond Natural Selection. London: Texere/Thomson, 2003

37 Margulis L. Origin of Eukaryotic Cells. New Haven: Yale University Press, 1970

38 Smith JM. A Darwinian view of symbiosis. In Margulis L, Fester R, eds. Symbiosis as a Source of Evolutionary Innovation. Cambridge, Mass: MIT Press, 1991

39 Bonnaud B, Bouton O, Oriel G, et al. Evidence of selection on the domesticated ERVWE1 env retroviral element involved in placenation. Mol Biol Evol (in press)

40 Boucher DH, ed. The Biology of Mutualism. New York: Oxford University Press, 1985

41 Villarreal LP, De Philippis VR. A hypothesis for DNA viruses as the origin of eukaryotic replication proteins. J Virol 2000;74:7079-84

42 Villarreal LP. Viruses and the Evolution of Life. Washington, DC: American Society for Microbiology (in press)

43 Lee X, Keith JC, Stumm N, et al. Downregulation of placental syncytin expression and abnormal protein localisation in pre-eclampsia. Placenta 2001;22:808-12

44 Knerr I, Beinder E, Rascher W. Syncytin, a novel human endogenous retroviral gene in human placenta: evidence for its dysregulation in pre-eclampsia and HELLP syndrome. Am $J$ Obstet Gynecol 2002;186:210-13

45 Knerr I, Weigel C, Linnemann K, et al. Transcriptional effects of hypoxia on fusiogenic syncytin and its receptor ASCT2 in human cytotrophoblast BeWo cells and in ex vivo perfused placental cotyledons. Am J Obstet Gynecol 2003;189:583-8

46 Knerr I, Huppertz B, Weigel C, et al. Endogenous retroviral syncytin: compilation of experimental research on syncytin and its possible role in normal and disturbed human placentogenesis. Molec Hum Reprod (in press) 